\title{
Diagnostik und Therapie von Lebermetastasen bei kolorektalem Primärtumor
}

\author{
Dirk Arnold $^{\mathrm{a}}$ Ernst J. Rummeny ${ }^{\mathrm{b}}$ Thomas Kirchner $^{\mathrm{c}} \quad$ Karsten Ridwelski $^{\mathrm{d}}$ \\ Helmut Oettle $e^{e, f}$ Jörg Thomas Hartmann ${ }^{g}$ Markus Moehler $^{\mathrm{h}}$ Arnulf Hölscher ${ }^{\mathrm{i}}$ \\ Hauke Lang ${ }^{j}$ Hans J. Schlitt ${ }^{k}$ Peter Neuhaus' \\ ${ }^{a}$ Klinik und Poliklinik für Innere Medizin IV, Abteilung für Onkologie und Hämatologie, Martin-Luther-Universität Halle-Wittenberg, \\ Halle (Saale); ${ }^{\mathrm{b}}$ Institut für Röntgendiagnostik, Klinikum rechts der Isar, Technische Universität München; ${ }^{\mathrm{c}}$ Pathologisches Institut, \\ Ludwig-Maximilians-Universität München; ${ }^{d}$ Klinik für Allgemein- und Viszeralchirurgie, Klinikum Magdeburg; ${ }^{\mathrm{e}}$ Charité Zentrum für \\ Tumormedizin, Berlin; ${ }^{f}$ Onkologische Schwerpunktpraxis, Friedrichshafen; ${ }^{g}$ Südwestdeutsches Tumorzentrum, Medizinische Klinik II, \\ Tübingen; ${ }^{\mathrm{h}}$ I. Medizinische Klinik und Poliklinik, Universitätsmedizin Mainz; ${ }^{\mathrm{i}}$ Klinik für Allgemein-, Viszeral- und Tumorchirurgie, \\ Universitätsklinik Köln; ${ }^{j}$ Klinik für Allgemein- und Abdominalchirurgie, Universitätsmedizin Mainz; ${ }^{k}$ Klinik und Poliklinik für Chirurgie, \\ Universitätsklinikum Regensburg; ' Klinik für Allgemein-, Viszeral- und Transplantationschirurgie, Charité Campus Virchow-Klinikum, \\ Universitätsmedizin Berlin, Deutschland
}

\section{Schlüsselwörter}

MSCT · DWI-MRT · Kolorektales Karzinom · LebermetastasenResektion · Neoadjuvante Therapie · Adjuvante Therapie ·

Radiofrequenz-Ablation

\section{Zusammenfassung}

Die kontrastverstärkte Mehrschicht-Computertomographie (MSCT) hat sich als das Standard-Schnittbildverfahren sowohl zur Diagnostik als auch zur Therapiekontrolle von Leberläsionen durchgesetzt. Zur Abklärung der lokalen Verhältnisse vor einer Leberteilresektion kann die diffusionsgewichtete Magnetresonanztomographie (DWI-MRT) wichtige zusätzliche Befunde liefern. Um das Kriterium der R0-Resektion zu erfüllen, erscheint ein Sicherheitsabstand von $0,5 \mathrm{~mm}$ ausreichend. Eine neoadjuvante Chemotherapie mit dem Ziel, die Tumorgröße zu verringern, kann parallel zur Pfortaderembolisation durchgeführt werden, ohne dass die perioperative Morbidität und Mortalität beeinträchtigt wird. Bezüglich des Managements primär resektabler Lebermetastasen besteht dringender Studienbedarf. Trotz der eher geringen Evidenz wird die adjuvante Chemotherapie in Deutschland derzeit breiter befürwortet als die perioperative Chemotherapie. Auch bezüglich der präoperativen Therapie bei Patienten mit (noch) nicht resektablen Lebermetastasen besteht erheblicher Studienbedarf. Bei KRAS-Wildtyp-Tumoren werden mit einer Cetuximab/Chemotherapie-Kombination hohe Ansprechraten (im Sinne einer metrischen Verkleinerung der Metastasen) erzielt. Bevacizumab/Chemotherapie-Kombinationen führen zu hohen pathohistologischen Komplett- und Partialremissionen. Welches der beste Parameter zur Erfolgsbeurteilung einer präoperativen Therapie ist, ist derzeit noch unbekannt, weshalb Vergleichsstudien mit dem Überleben als hartem Endpunkt durchgeführt werden müssen.
Key Words

MSCT · DWI-MRT · Colorectal cancer · Liver metastases resection - Neoadjuvant therapy · Adjuvant therapy ·

Radiofrequency ablation

\section{Summary}

Diagnosis and Treatment of Liver Metastases from Primary Colorectal Tumour

Contrast-enhanced multislice computer tomography (MSCT) has established itself as the standard tomographic imaging method both for diagnosis and for treatment monitoring of hepatic lesions. To clarify local conditions before partial liver resection, diffusion-weighted magnetic resonance tomography (DWI-MRT) can also provide important additional information. In order to meet the criteria for a R0 resection, a margin of $0.5 \mathrm{~mm}$ seems to be sufficient. Neoadjuvant chemotherapy aiming to reduce tumour size can be given in parallel with portal artery embolisation without adversely affecting perioperative morbidity and mortality. As far as the management of primary resectable liver metastases is concerned, there is an urgent need for more studies. Despite the relatively limited evidence, adjuvant chemotherapy is currently more widely favoured in Germany than perioperative chemotherapy. There is also considerable need for studies concerning preoperative therapy in patients with liver metastases that are not (yet) resectable. In KRAS wild-type tumours, high response rates (in terms of a reduction in the size of metastases) are achieved with a cetuximab/chemotherapy combination. Bevacizumab/ chemotherapy combinations lead to high rates of pathohistological complete and partial remissions. What the best parameter for judging the success of preoperative therapy is remains unknown, and so comparison studies using survival as a 'hard' endpoint must be carried out. 
Standards und neue Möglichkeiten bei der Detektion und dem Therapiemonitoring: Bedeutung von kontrastverstärktem Ultraschall, CT, MRT und PET-CT

Die derzeit gültigen Standards der Bildgebung beim kolorektalen Karzinom sind in der 2004 im Volltext erschienenen S3-Leitlinie festgelegt [1], bedürfen angesichts der rasanten Entwicklung der bildgebenden Verfahren in den letzten Jahren aber einer dringenden Überarbeitung.

Während die Ultraschalldiagnostik in der Hand des Geübten, insbesondere nach intravenöser Gabe von Ultraschallkontrastmitteln, eine sehr gute Erkennbarkeit und Differenzierung von Leberläsionen erlaubt, hat sich die kontrastverstärkte Mehrschicht-Computertomographie (MSCT) als das Standard-Schnittbildverfahren sowohl zur Diagnostik als auch zur Therapiekontrolle durchgesetzt. Bei fraglichen Leberläsionen und zur genaueren Abklärung der lokalen Verhältnisse vor einer Leberteilresektion kann die Magnetresonanztomographie (MRT) wichtige zusätzliche Befunde liefern. So erlaubt dieses Verfahren bei Einsatz spezieller Pulssequenzen wie der diffusionsgewichteten Bildgebung (DWI) oder bei Verwendung von leberspezifischen Kontrastmitteln (Primovist $^{\circledR}$, Endorem $\left.{ }^{\circledR}\right)$ eine optimale Detektion auch kleinster Läsionen [2]. Hierbei ist die DWI-MRT allen anderen nichtinvasiven bildgebenden Verfahren, einschließlich der CT und PET-CT (Positronenemissionstomographie), deutlich überlegen [2a, 2b].

Auch der lokale Befund lässt sich beim Kolon- oder Rektumkarzinom mit der CT und der MRT sehr gut darstellen. Die Leitlinie führt hierzu aus: «Ist aus technischen Gründen eine komplette Koloskopie nicht möglich, sollte als alternatives Verfahren z.B. die CT- oder MRT-Koloskopie eingesetzt werden». Bei Betrachtung der Meta-Analysen aus dem Jahr 2005 an insgesamt 6393 Patienten zeigt sich, dass die CT-Koloskopie für Polypen und Tumoren $>10 \mathrm{~mm}$ eine Sensitivität von $85-93 \%$ und eine Spezifität von $97 \%$ aufweist. Polypen mit einer Größe zwischen 6 und 9 mm werden mit einer Sensitivität von $70-86 \%$ bei einer Spezifität von 86-93\% erkannt [3].

Die Indikation zur PET-CT ergibt sich bei Verdacht auf extrahepatische Befunde dann, wenn eine Ganzkörper-Diagnostik gewünscht wird. So konnte in verschiedenen Studien gezeigt werden, dass mit der PET-CT okkulte entfernte Lymphknotenmetastasen sehr gut erkannt werden können.

Zur Beurteilung des Therapieerfolges spielt die PET-CT im Bereich der Leber bisher keine Rolle. Hier sind als Schnittbildverfahren insbesondere die CT und MRT (Abb. 1, 2) gefordert. Beide Verfahren beziehen zur Abschätzung eines Therapieerfolges GröBenmessungen ein, wobei die Auswertung dann im Weiteren nach RECIST-Kriterien erfolgt. Hierzu werden heute vorwiegend 2D-Messungen durchgeführt. Erste Studien belegen jedoch, dass 3D-Messungen aussagekräftiger sein können.

Die neuen Therapieverfahren mit Antikörper/ChemotherapieKombinationen zeigen aufgrund ihres Wirkmechanismus teilweise andere schnittbildmorphologische Veränderungen als die Chemotherapie. Solche Phänomene sind z. B. zystische Umbildungen des Tumors bei unveränderter Größe oder pathohistologische Komplettremissionen, obwohl kaum eine Größenänderung stattgefunden hat. Somit stößt die Beurteilung des Therapieerfolges nach den derzeitigen RECIST-Kriterien hier an ihre Grenzen. Erkennt-

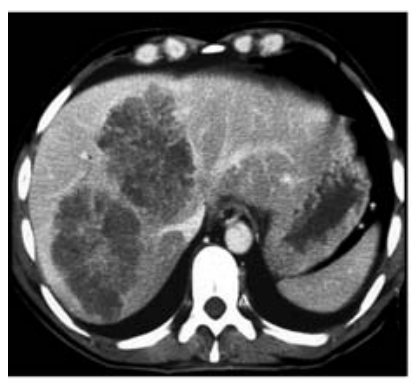

vor CTx

Abb. 1. Metastasen bei Kolon-Ca.

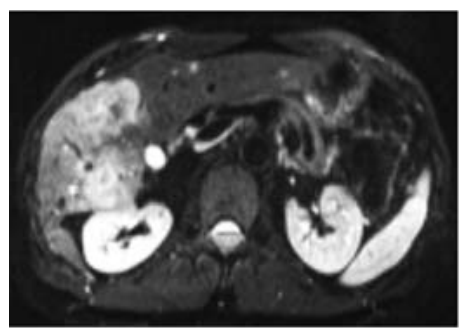

Initiales MRT

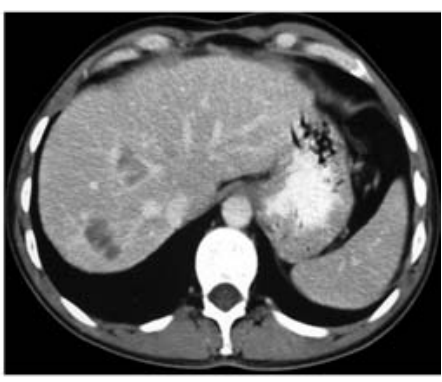

nach CTx

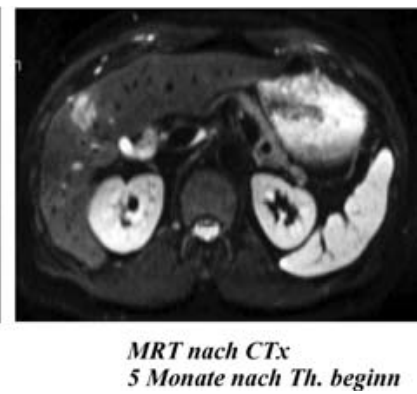

Abb. 2. Metastasen bei Rektum-Ca

nisse, die diese «biologischen» Therapiephänomene besser charakterisieren, erhofft man sich durch die Verwendung von «funktionellen» Informationen, z. B. von Perfusionsstudien bei der CT. Bei der MRT scheint sich mit Hilfe der Diffusionsbildgebung ein neuer messbarer Parameter, der Diffusionskoeffizient, abzuzeichnen. Erste Untersuchungen zeigen, dass dieser Wert bei erfolgreicher Therapie deutlich ansteigt. Auch die PET-CT könnte hier als funktionelles Verfahren wichtige Beiträge leisten, allerdings sind zum jetzigen Zeitpunkt entsprechende Daten noch sehr spärlich. Lediglich zur lokalen Therapieerfolgskontrolle des Rektumkarzinoms liegen einige Arbeiten vor, die eine deutliche Abnahme der Tracer-Aufnahme bei Respondern bereits 14 Tage nach Therapiebeginn zeigen [4].

\section{Lebermetastasen - Epidemiologie und Klassifizierung}

Jedes Jahr erkranken in Deutschland etwa 72000 Menschen an einem kolorektalen Karzinom. Bei 10-20\% der Betroffenen liegen synchrone oder metachrone Lebermetastasen vor, ohne dass extrahepatische Fernmetastasen festgestellt werden. Diese 7000-14 000 Patienten pro Jahr sind möglicherweise heilbar. Denn die 5-JahresÜberlebensrate nach der Resektion von Lebermetastasen liegt derzeit bei etwa $40 \%$ (Abb. 3) - und könnte durch eine verbesserte präoperative Diagnostik, neue chirurgische Verfahren und medikamentöse Therapien in Zukunft noch deutlich ansteigen [5]. Grundlegende Voraussetzung für die Evaluierung neuer Therapieprinzipien in klinischen Studien ist aber eine prognostisch relevante und international anerkannte Klassifikation der Lebermetastasierung. Darüber hinaus muss einheitlich definiert werden, wann Lebermetastasen resektabel bzw. kurabel sind und wann nicht. 


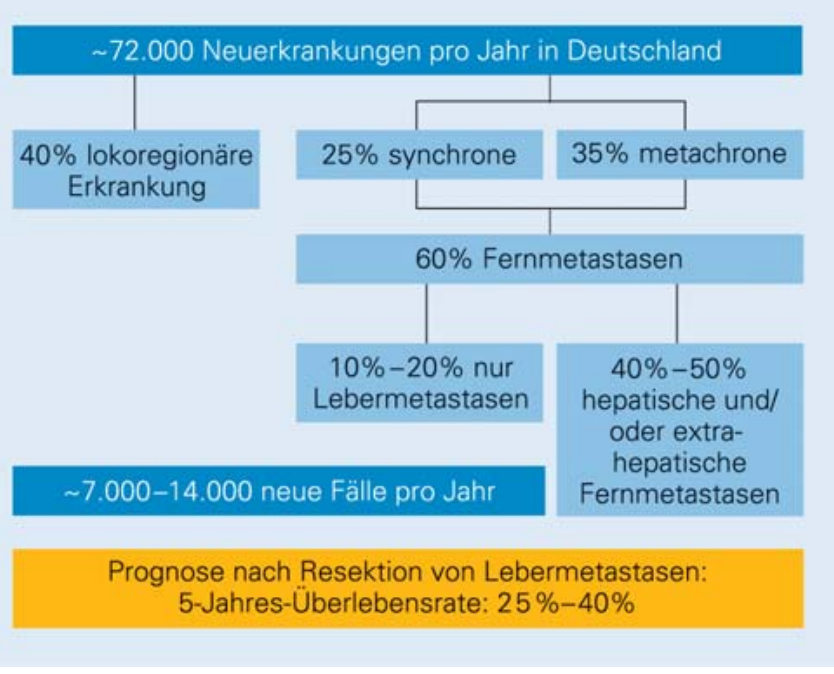

Abb. 3. Kolorektales Karzinom - Neuerkrankungen in Deutschland pro Jahr.

Vorschläge zur Lösung des Problems wurden kürzlich publiziert [6-10], ein Konsensus liegt aber noch nicht vor.

\section{Chirurgische Verfahren zur Verbesserung der Resektabilität}

Wichtige Kriterien der Resektabilität sind die Lokalisation und die Zahl der Metastasen sowie insbesondere das Volumen des funktionsfähigen Restparenchyms. Früher bedeuteten $>3$ Lebermetastasen, ein zu erwartender Sicherheitsabstand von $<1 \mathrm{~cm}$ zum gesunden Lebergewebe und ein zu erwartendes Restparenchym von $<40 \%$ Irresektabilität.

Heute erscheint selbst ein Sicherheitsabstand von $0,5 \mathrm{~mm}$ ausreichend, um das Kriterium der R0-Resektion zu erfüllen [5], denn die Prognose dieser Patienten ist nicht schlechter als die von Patienten mit größerem Sicherheitsabstand [11]. Bei optimalem technischem Vorgehen lassen sich selbst bei R1-resezierten Patienten Langzeitüberlebenszeiten erreichen, die denen von R0-resezierten Patienten kaum nachstehen. So publizierten de Haas et al. [12] kürzlich 10-Jahres-Überlebenszeiten von $43 \%$ bei R0-resezierten Patienten und von $37 \%$ bei R1-resezierten Patienten.

Patienten mit mehr als 3 Lebermetastasen haben nach retrospektiven Analysen zwar eine signifikant schlechtere Prognose als Patienten mit weniger Metastasen [5, 13], sie gelten aber keineswegs grundsätzlich als nicht operabel. Entscheidend ist, dass 2 benachbarte Lebersegmente erhalten bleiben und dass das zu erwartende funktionsfähige Restparenchym $>20 \%$ beträgt, wenn das Lebergewebe nicht vorgeschädigt ist. Nach einer Chemotherapie sollte das zu erwartende funktionsfähige Restparenchym bei $>30-40 \%$ liegen. Sind diese Volumina primär nicht zu erreichen, ist eine Pfortaderembolisation (PVE) oder eine Pfortaderligatur indiziert. Diese sollte deutlich vor dem geplanten Eingriff durchgeführt werden und kann das zu erwartende Restvolumen erheblich steigern. Alternativ ist auch eine zweizeitige Leberresektion möglich. Eine neoadjuvante Chemotherapie kann parallel zur PVE durchgeführt werden, ohne dass die perioperative Morbidität und Mortalität beeinträchtigt wird [14] (Abb. 4).

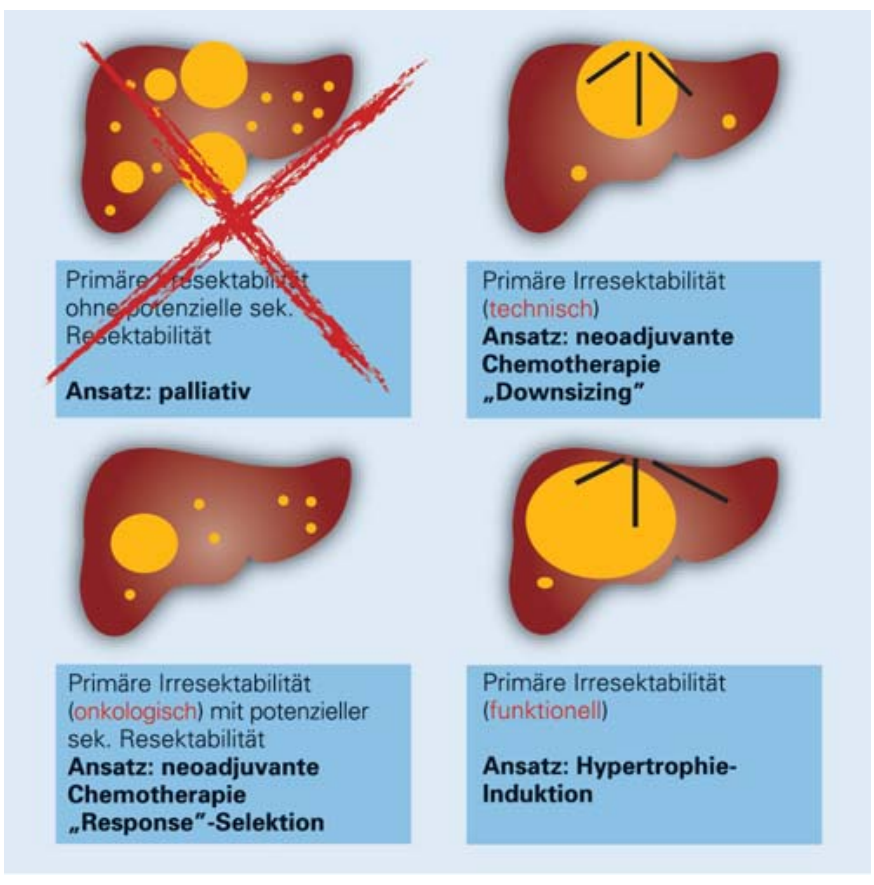

Abb. 4. Primäre Irresektabilität - welcher Ansatz?

\section{Bedeutung der kompletten radiologischen Response}

Sprechen Lebermetastasen auf eine neoadjuvante Chemotherapie vollständig an, bedeutet das in den meisten Fällen dennoch keine Heilung. So waren in einer Studie von 66 radiologisch nicht mehr nachweisbaren Lebermetastasen intraoperativ 20 dennoch sichtbar. Von den restlichen 46, die makroskopisch nicht mehr erkennbar waren, wurde ein Teil reseziert, ein Teil belassen. In $80 \%$ der Resektate waren vitale Tumorzellen vorhanden. An den nicht resezierten Stellen traten in 74 \% der Fälle Lokalrezidive auf [15].

Lebermetastasen sollten deshalb möglichst vor dem kompletten Ansprechen reseziert werden, einerseits, um intraoperativ gut erkennbar zu sein, und andererseits, um die Patienten nicht unnötig lange mit potenziell toxischen Chemotherapeutika zu belasten. Wenn die Metastasen nicht mehr sichtbar sind, muss die Region dennoch mitreseziert werden, um Lokalrezidive zu verhindern. Ungelöst ist das Problem, wie eine befallene Region trotz Vollremission sicher wiederzufinden ist.

\section{Radiofrequenz-Ablation}

Lokoregionäre Therapieverfahren wie die Radiofrequenz-Ablation (RFA) oder die Laser-induzierte interstitielle Thermotherapie (LITT) werden zum einen ergänzend zur chirurgischen Intervention eingesetzt, um bei irresektablen oder nicht R0-resektablen Metastasen das chirurgische Ergebnis zu verbessern und möglichst doch «Tumorfreiheit» zu erreichen. Zum anderen können diese Verfahren bei ausgewählten Patienten, bei denen eine Resektion nicht möglich ist, auch allein zum Einsatz kommen. Ein Hauptproblem dieser Verfahren ist aber die hohe Lokalrezidivrate. Je kleiner die Metastase, desto eher gelingt eine vollständige Thermoablation (R0-Ablation). Ab einer Größe von $>3 \mathrm{~cm}$ steigt die Rezidivrate deutlich an [16]. 
Die perkutane RFA führt selbst bei kleinen Metastasen zu einer deutlich höheren Lokalrezidivrate als die im Rahmen einer Laparoskopie oder Laparotomie durchgeführte RFA. Deshalb ist der chirurgische Weg für Patienten, die eine Laparoskopie oder Laparotomie tolerieren können, die erste Wahl $[16,17]$.

\section{Primär resektable Lebermetastasen - prä-/postoperative oder (keine) adjuvante Therapie?}

Ob Patienten mit resektablen Lebermetastasen eine neoadjuvante und/oder adjuvante Chemotherapie erhalten sollen, wird nach wie vor kontrovers diskutiert. Denn die klinischen Studien, die hierzu in den vergangenen Jahren publiziert wurden, geben keine definitive Antwort und lassen unterschiedliche Interpretationen zu.

Ist eine R0-Resektion möglich (primär oder nach Vorbehandlung), sollte diese aus chirurgischer Sicht ohne Verzögerung erfolgen. Aus internistischer Sicht wird auf die EORTC-Studie 40983 verwiesen, die zeigen konnte, dass die perioperative (also prä- und postoperative) Chemotherapie mit dem FOLFOX4-Schema (infusionales 5-FU/FS + Oxalipatin) bei Patienten mit primär resektablen Lebermetastasen das Rezidivrisiko um $19 \%$ reduziert. Das 3-Jahres-progressionsfreie Überleben konnte im Vergleich zu den nur resezierten Patienten um absolut 7,3\% (von 28,1 auf 35,4\%; ITT-Analyse) angehoben werden - dies bei einer Patientengruppe, die aufgrund ihrer Metastasierungscharakteristika mit im Median nur einer solitären Metastase «ideale» Kandidaten für eine primäre Resektion gewesen wären. Der Unterschied von 7,3\% verfehlte in der ITT-Analyse, dem primären Studienendpunkt, zwar knapp das Signifikanzniveau $(\mathrm{p}=0,058)$. In der Analyse der «eligible patients» und der «resected patients», also der auswertbaren Patienten und derjenigen, die tatsächlich reseziert wurden, wurde das Signifikanzniveau aber erreicht ( $p=0,041$ bzw. 0,025) (Tab. 1) [18]. Vor diesem Hintergrund wird die jeweils 3-monatige prä- und postoperative Chemotherapie mit FOLFOX von der durchführenden Studiengruppe EORTC als der neue Standard empfohlen.

Eine Alternative stellt die postoperative (adjuvante) Chemotherapie dar. Allerdings ist die Evidenzlage auch hierfür unbefriedigend: Zwei Phase-III-Studien, in denen ein heute veraltetes 5-FUBolus-Regime eingesetzt wurde, mussten wegen unzureichender Rekrutierung vorzeitig abgebrochen werden $[19,20]$. In der Studie von Portier et al. [19] konnte zu diesem Zeitpunkt eine signifikante
Verlängerung des 5-Jahre-progressionsfreien-Überlebens gezeigt werden, wenn die Patienten nach der Leberresektion adjuvant mit 5-FU behandelt wurden (26,7 vs. $33,5 \%$; $=0,028)$. Die gepoolte Analyse der Daten beider abgebrochener Studien kommt hingegen zu keiner signifikanten Verbesserung des Überlebens, wenngleich das mediane Gesamtüberleben in der Therapiegruppe bei 62,2 Monaten lag - gegenüber 47,3 Monaten in der Kontrollgruppe [21]. Zwei aktuellere Studien, die eine adjuvante KombinationsChemotherapie prüften (ADHOC, NSABP C09) mussten ebenfalls wegen unzureichender Rekrutierung abgebrochen werden. Eine große Registerstudie konnte bei Patienten, die nach der Leberresektion eine adjuvante 5-FU-basierte Therapie erhielten, einen signifikanten Überlebensvorteil zeigen $(p=0,007)$ und unterstützt somit die Strategie der adjuvanten Therapie nach Metastasenresektion [22].

Aufgrund dieser Daten wird in den deutschen Leitlinien die adjuvante Therapie prinzipiell befürwortet mit dem Wortlaut, dass «nach R0-Resektion synchroner oder metachroner Lebermetastasen eine adjuvante Chemotherapie erwogen werden kann» (Evidenzgrad 2, Empfehlungsgrad B) [23]. Eine «neoadjuvante Therapie resektabler Lebermetastasen kann in begründeten Ausnahmefällen erwogen werden» (Evidenzgrad 3, Empfehlungsgrad 0) [23]. Weitere Hintergründe für die Präferenz der adjuvanten Therapie in Deutschland sind zum einen das Risiko der Leberschädigung durch die neoadjuvante Chemotherapie und damit der potenziellen Inoperabilität, zum anderen das Risiko des Nichtansprechens auf die neoadjuvante Chemotherapie, wodurch Patienten möglicherweise ebenfalls inoperabel werden könnten.

\section{Wen behandeln und womit?}

Ein nicht geklärtes Problem ist, welche Patienten tatsächlich von einer begleitenden Chemotherapie profitieren. Zum Teil wird die Ansicht vertreten, dass Patienten mit einem höheren Risiko für ein Tumorrezidiv (Fong-Score > III [24]) den größten Nutzen haben. Hierfür gibt es jedoch wiederum keine ausreichende Evidenz. Allerdings hat die randomisierte Studie von Portier et al. [19] gezeigt, dass Patienten mit größerer Tumorlast (multiple Metastasen oder $>5 \mathrm{~cm}$ ) einen Überlebensvorteil aufweisen, wenn sie adjuvant behandelt werden. Andererseits weisen Studien beim Rektumkarzinom darauf hin, dass gerade die Patienten mit niedrigem Risiko

Tab. 1. Perioperative Chemotherapie mit FOLFOX bei resektablen Lebermetastasen (nach: Nordlinger B et al., Lancet 2008;371(9617):1007-1016)

\begin{tabular}{|c|c|c|c|c|c|}
\hline & $\begin{array}{l}\text { Anzahl der } \\
\text { Patienten mit } \\
\text { Chemotherapie }\end{array}$ & $\begin{array}{l}\text { Anzahl der } \\
\text { Patienten mit } \\
\text { Chirurgie }\end{array}$ & $\begin{array}{l}\text { absoluter Unterschied } \\
\text { im 3-Jahres-progressionsfreien } \\
\text { Überleben }(\%)\end{array}$ & $\begin{array}{l}\text { Hazard Ratio } \\
\text { (95\%-Konfidenz- } \\
\text { intervall) }\end{array}$ & p-Wert \\
\hline alle Patienten & 182 & 182 & $\begin{array}{l}\mathbf{+ 7 , 3} \% \\
\text { (Steigerung von } 28,1 \% \text { auf } \\
35,4 \% \text { ) }\end{array}$ & $\begin{array}{l}\mathbf{0 , 7 9} \\
(0,62-1,02)\end{array}$ & $\mathrm{p}=0,058$ \\
\hline $\begin{array}{l}\text { alle auswertbaren } \\
\text { Patienten }\end{array}$ & 171 & 171 & $\begin{array}{l}\mathbf{+ 8 , 1} \% \\
\text { (Steigerung von } 28,1 \% \text { auf } \\
36,2 \% \text { ) }\end{array}$ & $\begin{array}{l}\mathbf{0 , 7 7} \\
(0,60-1,00)\end{array}$ & $\mathrm{p}=0,041$ \\
\hline $\begin{array}{l}\text { alle resezierten } \\
\text { Patienten }\end{array}$ & 151 & 152 & $\begin{array}{l}\mathbf{+ 9 , 2} \% \\
\text { (Steigerung von } 33,2 \% \text { auf } \\
42,4 \% \text { ) }\end{array}$ & $\begin{array}{l}\mathbf{0 , 7 3} \\
(0,55-0,97)\end{array}$ & $\mathrm{p}=0,025$ \\
\hline
\end{tabular}


von einer neoadjuvanten Therapie besonders profitierten [25-27]. Entscheidet man sich für eine neoadjuvante Behandlung, sollte eine Kombinations-Chemotherapie erfolgen - eventuell mit der zusätzlichen Gabe einer molekularen Substanz, wie es in verschiedenen Studienkonzepten geprüft worden ist, ohne dass ein erhöhtes Risiko für perioperative Komplikationen beobachtet wurde $[28,29]$. Eine 5-FU/FS-Monotherapie ist aufgrund der publizierten Daten in der adjuvanten Therapie zu begründen. Auch postoperativ besteht eine Rationale für die Kombinations-Chemotherapie. Wird neoadjuvant behandelt, sollte nach 4 Wochen eine Reevaluation erfolgen, um das Risiko der Inoperabilität wegen Nichtansprechens auf die neoadjuvante Therapie möglichst gering zu halten. Ist der Tumor progredient, aber noch operabel, sollte in jedem Falle sofort operiert werden, denn auch in diesen Fällen werden gute Langzeitergebnisse erzielt [30].

Einigkeit besteht von chirurgischer und internistischer Seite darin, dass bezüglich des Managements resezierbarer Lebermetastasen dringender Studienbedarf besteht, weshalb jetzt die gemeinsame Planung und Durchführung einer deutschlandweiten Studie in die Wege geleitet werden soll.

\section{«Konversionstherapie» - was wie lange geben?}

Welche präoperative Therapie für Patienten mit nicht resektablen oder «grenzwertig» resektablen Lebermetastasen die beste ist, lässt sich derzeit nicht klar beantworten, so dass auch hier erheblicher Studienbedarf besteht. Ansprechraten und Resektionsraten aus verschiedenen Studien miteinander zu vergleichen («cross-trial comparison»), führt aufgrund der Variabilität der Kriterien zu keiner zuverlässigen Aussage.

Grundsätzlich lässt sich jedoch sagen, dass Dreifach-Kombinationen (fast) alle zu höheren Ansprechraten führen als ZweifachKombinationen und dass hohe Ansprechraten offenbar mit hohen Resektionsraten korrelieren [31].

Bis vor kurzem war unklar, wie hoch die «Konversionsrate» hin zur Resektabilität unter Verwendung einer sehr aktiven Chemotherapie tatsächlich ist. Prospektiv untersucht wurde dies in der randomisierten CELIM-Studie, in der Patienten mit nicht resektablen Lebermetastasen in neoadjuvanter Intention entweder FOLFOX oder FOLFIRI und zusätzlich Cetuximab erhielten. Bei Patienten mit einem KRAS-Wildtyp-Tumor ließ sich eine Gesamtansprechrate von $70 \%$ dokumentieren. Im Gesamtkollektiv wurde eine R0-Resektionsrate von $34 \%$ erreicht [32]. Interessant ist, dass $25 \%$ der initial als irresektabel eingeschätzten Patienten in einem verblindeten zentralen Review-Verfahren als durch den Therapieerfolg zur Resektabilität konvertiert beurteilt wurden.

Auch für andere Kombinationen wie die Dreifach-Chemotherapie mit FOLFOXIRI (5-FU/Oxaliplatin/Irinotecan) wurden hohe Ansprechraten berichtet [33], desgleichen für die Vierfach-Therapie FOLFOXIRI + Bevacizumab [34] oder FOLFOXIRI + Cetuximab [35]. Allerdings ist noch nicht bewiesen, dass hohe Ansprechraten, also die metrische Verkleinerung von Metastasen, letztlich auch der beste Parameter zur Erfolgsabschätzung des präoperativen Therapieerfolges sind und zu verbesserten Überlebenszeiten führen: Möglicherweise liegt in der «biologischen» Aktivität, z. B. ausgedrückt durch die Reduktion des Anteils von viablen («lebendigen») Tumorzellen, ein größerer Nutzen für das Gesamtüberleben als in einer Response nach RECIST-Kriterien.
In diesem Zusammenhang konnte gezeigt werden, dass in den resezierten Metastasen von mit Bevacizumab und einer Oxaliplatinhaltigen Chemotherapie vorbehandelten Patienten signifikant weniger viable Tumorzellen vorhanden waren als bei den Patienten, die nur eine Chemotherapie erhalten hatten [36]. Diese Daten wurden durch eine aktuelle Analyse der Resektionspräparate von insgesamt 219 Patienten nach Lebermetastasenresektion bestätigt [37]. Die Patienten hatten präoperativ entweder Bevacizumab plus FOLFOX oder nur FOLFOX erhalten. Es zeigte sich, dass die Patienten mit Bevacizumab-haltiger präoperativer Therapie eine höhere Rate an pathohistologischen Komplett- und Partialremissionen aufwiesen (Abb. 5). Andere Arbeiten haben berichtet, dass gerade Patienten mit einer kompletten pathohistologischen Remission sehr gute Überlebensdaten haben, weshalb die komplette pathohistologische Remission als ein möglicher Endpunkt für den Erfolg einer präoperativen Therapie diskutiert wird (Abb. 6) [38].

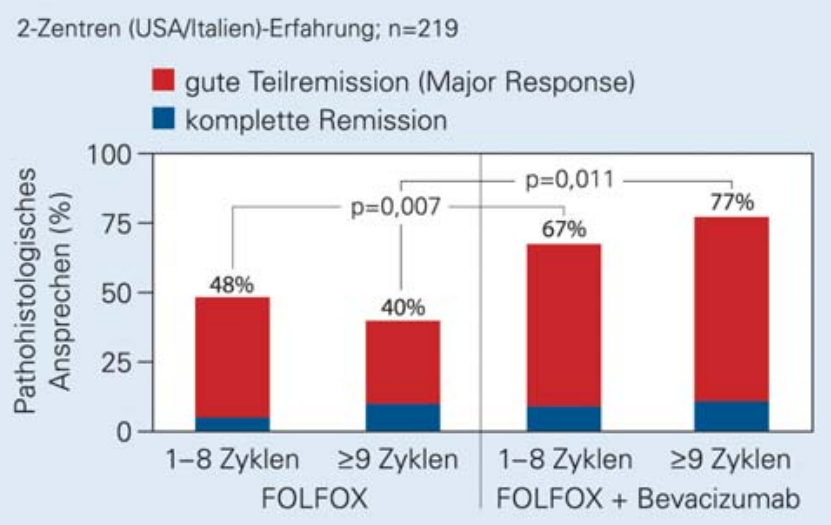

Abb. 5. Signifikanter Anstieg der Pathohistologischen Komplett- und Partialremissionen unter der Präoperativen Bevacizumab-haltigen Therapie (nach: Zorzi D et al., ASCO GI 2009, Abstract 295).

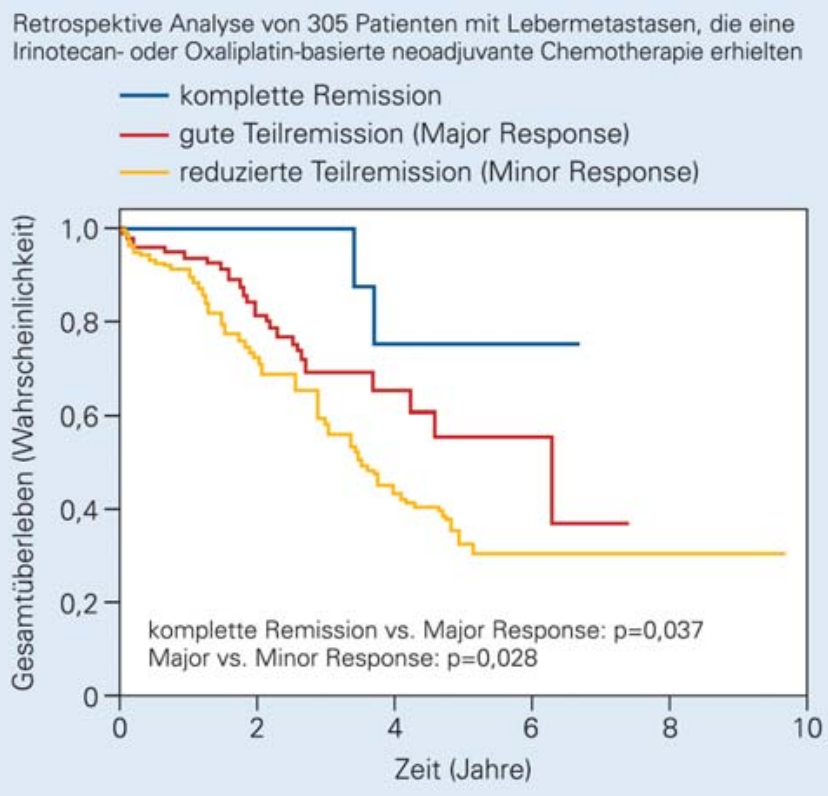

Abb. 6. Pathohistologisches Ansprechen auf die präoperative Chemotherapie: ein neuer Endpunkt nach der Resektion von kolorektalen Lebermetastasen? (nach: Blazer DG et al., J. Clin Oncol 2008;26(33):5344-5351). 
Bezüglich der Toxizität gilt, dass alle derzeit verfügbaren DreifachKombinationen «machbar» sind, wobei die Bevacizumab-Chemotherapie-Kombination die beste subjektive Verträglichkeit aufweist. Perioperative Komplikationen sind durch Bevacizumab offenbar nicht erhöht [28, 29], und in Bezug auf die Leber weist Bevacizumab eher eine protektive Wirkung auf $[36,37]$ und steigert die Leberinsuffizienzrate nicht.

Was die Dauer der präoperativen Therapie angeht, so konnte gezeigt werden, dass die Leberinsuffizienzrate signifikant ansteigt, wenn präoperativ $>8$ Zyklen Chemotherapie verabreicht werden [37]. Deshalb sollte die Therapie in der Regel 4 Monate nicht überschreiten.

\section{Disclosure Statement}

The authors have been speakers and contributors to the meeting and the publication "IX. Interdisziplinärer Expertenworkshop "Gastrointestinale Tumore', Schlossgut Oberambach, Münsing”, sponsored by Roche Pharma AG.

\section{Literatur}

1 Schmiegel W, et al: S3-Guidelines Conference "Colorectal Carcinoma" 2004. Z Gastroenterol 2004;42:1129-1177.

2 Hammerstingl R, et al: Diagnostic efficacy of gadoxetic acid (Primovist)-enhanced MRI and spiral CT for a therapeutic strategy: comparison with intraoperative and histopathologic findings in focal liver lesions. Eur Radiol 2008;18:457-467.

2a Bruegel M, et al: Diagnosis of hepatic metastasis: comparison of respiration-triggered diffusionweighted echo-planar MRI and five T2-weighted turbo spin-echo sequences. AJR Am J Roentgenol 2008;191:1421-1429.

$2 \mathrm{~b}$ Bruegel M, et al: Characterization of focal liver lesions by ADC measurements using a respiratory-triggered diffusion-weighted single-shot echo-planar MR imaging technique. Eur Radiol 2008;18:477-485.

3 Halligan S, et al: CT colonography in the detection of colorectal polyps and cancer: systematic review, meta-analysis, and proposed minimum data set for study level reporting. Radiology 2005;237:893-904

4 Rosenberg R, et al: The predictive value of metabolic response to preoperative radiochemother apy in locally advanced rectal cancer measured by PET/CT. Int J Colorect Dis 2009;24:191-200.

5 Jonas S, et al: Extended resections of liver metastases from colorectal cancer. World J Surg 2007; 31:511-521.

6 Poston GJ, et al: Urgent need for a new staging system in advanced colorectal cancer. J Clin Oncol 2008;26:4828-4833.

7 Van Cutsem E, et al: Towards a pan-European consensus on the treatment of patients with colorectal liver metastases. Eur J Cancer 2006;42 2212-2221.

8 Yamaguchi T, et al: A new classification system for liver metastases from colorectal cancer in Japanese multicenter analysis. Hepatogastroenterology 2008;55:173-178.

9 Nagashima I, et al: Proposal of a new and simple staging system of colorectal liver metastasis. World J Gastroenterol 2006;12:6961-6965.

10 Are C, et al: The impact of margins on outcome after hepatic resection for colorectal metastasis. Ann Surg 2007;246:295-300.

11 Pawlik TM, et al: Effect of surgical margin status on survival and site of recurrence after hepatic resection for colorectal metastases. Ann Surg 2005;241:715-724.

12 de Haas RJ, et al: R1 resection by necessity for colorectal liver metastases: is it still a contraindication to surgery? Ann Surg 2008;248:626-637.

13 Figueras J, et al: Surgical resection of colorecta liver metastases in patients with expanded indications: a single-center experience with 501 patients. Dis Colon Rectum 2007;50:478-488.
14 Covey AM, et al: Combined portal vein embolization and neoadjuvant chemotherapy as a treatment strategy for resectable hepatic colorectal metastases. Ann Surg 2008;247:451-455.

15 Benoist S, et al: Complete response of colorectal liver metastases after chemotherapy: does it mean cure? J Clin Oncol 2006;24:3939-3945.

16 Mulier S, et al: Local recurrence after hepatic radiofrequency coagulation: multivariate metaanalysis and review of contributing factors. Ann Surg 2005;242:158-171.

17 Eisele RM, et al: Open surgical is superior to percutaneous access for radiofrequency ablation of hepatic metastases. World J Surg 2009;33:804811

18 Nordlinger B, Sorbye H, Glimelius B, et al: Perioperative chemotherapy with FOLFOX4 and surgery versus surgery alone for resectable liver metastases from colorectal cancer (EORTC Intergroup trial 40983): a randomised controlled trial. Lancet 2008;371:1007-1016.

19 Portier G, et al: Multicenter randomized trial of adjuvant fluorouracil and folinic acid compared with surgery alone after resection of colorectal liver metastases: FFCD ACHBTH AURC 9002 trial. J Clin Oncol 2006;24:4976-4982.

20 Langer B, et al: Fluorouracil (FU) plus l-leucovorin (l-LV) versus observation after potentially curative resection of liver or lung metastases from colorectal cancer (CRC): results of the ENG (EORTC/NCIC CTG/GIVIO) randomized trial. Proc Am Soc Clin Oncol 2002;21:abstr 592.

21 Mitry E, et al: Adjuvant chemotherapy after potentially curative resection of metastases from colorectal cancer: a pooled analysis of two randomized trials. J Clin Oncol 2008;26:4906-4911.

22 Parks R, et al: Adjuvant chemotherapy improves survival after resection of hepatic colorectal metastases: analysis of data from two continents. J Am Coll Surg 2007;204:753-761.

23 Schmiegel W, et al: S3-Leitlinie «Kolorektales Karzinom»-Aktualisierung 2008. Z Gastroenterol 2008;46:799-840.

24 Fong Y, Fortner J, Sun RL, Brennan MF, Blumgart LH: Clinical score for predicting recurrence after hepatic resection for metastatic colorectal cancer: analysis of 1001 consecutive cases. Ann Surg 1999;230:309-318; discussion 318-321.

25 Sebag-Montefiore D, et al: Routine short course pre-op radiotherapy or selective post-op chemoradiotherapy for resectable rectal cancer? Preliminary results of the MRC CR07 randomised trial. ASCO 2006:abstr 3511.

26 Peeters KC, et al: The TME trial after a median follow-up of 6 years: increased local control but no survival benefit in irradiated patients with resectable rectal carcinoma. Ann Surg 2007;246: 693-701.
27 Marijnen CA, personal communication.

28 Grünberger B, et al: Bevacizumab, capecitabine, and oxaliplatin as neoadjuvant therapy for patients with potentially curable metastatic colorectal cancer. J Clin Oncol 2008;26:1830-1835.

29 Kesmodel SB, et al: Complication rates following hepatic surgery in patients receiving neoadjuvant bevacizumab (BV) for colorectal cancer (CRC) liver metastases. ASCO GI 2007:abstr 234.

30 Neumann UP, et al: Is chemotherapy failure a contraindication for liver resection in liver metastases of colorectal cancer? A multivariate analysis. ASCO 2007; abstr 4066, submitted to Surgery.

31 Folprecht G, et al: Neoadjuvant treatment of unresectable colorectal liver metastases: correlation between tumour response and resection rates. Ann Oncol 2005;16:1311-1319.

32 Folprecht G, et al: Cetuximab plus FOLFOX6 or cetuximab plus FOLFIRI as neoadjuvant treatment of nonresectable colorectal liver metastases: a randomized multicenter study (CELIM study). ASCO GI 2009;abstr 296

33 Falcone A, et al. for the Gruppo Oncologico Nord Ovest: Phase III trial of infusional fluorouracil, leucovorin, oxaliplatin, and irinotecan (FOLFOXIRI) compared with infusional fluorouracil, leucovorin, and irinotecan (FOLFIRI) as first-line treatment for metastatic colorectal cancer. J Clin Oncol 2007;25:1670-1676

34 Falcone A, et al: FOLFOXIRI (irinotecan, oxaliplatin, and infusional 5FU/LV) in combination with bevacizumab (BV) in the first-line treatment of metastatic colorectal cancer (mCRC): a phase II study by the G.O.N.O. group. ASCO 2008; abstr 4031

35 Samalin E, et al: Interim analysis of a multicenter phase II trial evaluating cetuximab in combination with FOLFIRINOX (LV5FU + irinotecan + oxaliplatin) as first-line treatment of metastatic colorectal cancer (mCRC) patients. ASCO GI 2008;abstr 375

36 Ribero D, et al: Bevacizumab improves pathologic response and protects against hepatic injury in patients treated with oxaliplatin-based chemotherapy for colorectal liver metastases. Cancer 2007;110:2761-2767.

37 Zorzi D, et al: Effect of extended preoperative chemotherapy on pathologic response and postoperative liver insufficiency after hepatic resection for colorectal liver metastases. ASCO GI 2009; abstr 295.

38 Blazer DG 3rd, et al: Pathologic response to preoperative chemotherapy: a new outcome end point after resection of hepatic colorectal metastases. J Clin Oncol 2008;26:5344-5351. 